\title{
Automatic Modulation Classification in Wireless Disaster Area Emergency Network (W-DAEN)
}

\author{
Md. Abdur Rahman, Azril Haniz, Minseok Kim and Jun-ichi Takada \\ Department of International Development Engineering, Graduate School of Science and Engineering \\ Tokyo Institute of Technology, Meguro, Tokyo 152-8552, Japan \\ Email: \{abdur, azril, mskim, takada\}@ap.ide.titech.ac.jp
}

\begin{abstract}
Post-disaster situation requires quick and effective rescue efforts by the first responders. Generally the rescue teams use wireless radios for intra-agency communications. Lack of collaboration among different rescue agencies may create interference among the emergency radios. Identification of some physical parameters of these active radios is necessary for collaboration. Carrier frequency and bandwidth can be estimated by spectrum sensing, whereas modulation classification requires further signal processing and classification operations. Processing speed and performance of the classification system can be controlled by appropriate selection of signal parameters, signal processing techniques and the classification algorithms. A wireless disaster area emergency network (W-DAEN) can be installed in the disaster area to detect and capture data (time samples) of the occupied frequencies. This study consists of some simulation results of a machine learning based cooperative automatic modulation classification technique by using six unique features. The classification performance and processing time of the proposed algorithm is quite satisfactory for real-time classification system.
\end{abstract}

\section{INTRODUCTION}

After big disasters (natural or manmade) generally a number of rescue teams come to the site in the first hour. In some countries emergency frequencies are pre-allocated for the first responders for better management. For example in Japan the emergency frequencies are allocated by different states to the local emergency responders. But for a big disaster rescue teams come from all over the world and setup their own wireless networks. At present most of the emergency radios are normally set to use pre-defined PHY parameters. In emergency situation, interferences occur when two or more networks try to use same carrier frequency for individual communications in close proximity. This interference among the corresponding radios is a big hindrance to the rescue operations. A central database that can collect, maintain and update the information of active emergency radio can help in such cases. Rescue teams may check the availability of corresponding frequencies before setting up their networks in the disaster area to avoid the interference. A conceptual architecture and description of Wireless Disaster Area Emergency Network (W-DAEN) is available in [1].

The organization of this paper is as follows. A brief background of the automatic modulation classification (AMC) is presented in section II. Introduction of the network architecture and system model is discussed in section III. Section IV con- sists of the methods of parameter extraction and classification algorithm. Simulation results are presented in section V while the concluding remarks are added in section VI.

\section{REVIEW OF AUTOMATIC MODULATION CLASSIFICATION}

Modulation classification techniques are mainly divided into two categories (in terms of used parameters), i) maximum likelihood and ii) feature based. Azzouz and Nandi [2] have proposed some features and algorithms to classify both analog and digital modulation signals. The features are derived from the instantaneous amplitude, frequency and phases of the received signals. References [3], [4] discussed about two classification approaches with the proposed features. The key features used by Azzouz and Nandi are later used by [5], [6] and many more. But in reality, accuracy of these features depend on some factors such as noise, carrier offset error, symbol rate estimation error that effect the success rate. Hossen et.al [7] also proposed an ANN based classification system by using the statistical signal characterization (SSC) parameters [8]. The SSC parameters are very good to observe the amplitude and frequency variations in the received signal. However, magnitude of the SSC parameters are sensitive to noise. So these parameters alone are not so useful in low SNR cases. In fact very few literature are available that can classify signals with very low SNR. Fortunately for the proposed scenario (disaster area) the sensors are expected to receive high SNR signal (because of the presence of line-of-sight). Effect of carrier frequency offset (CFO) of the receiver is another issue that will effect the accuracy of the SSC calculations. Moreover, SSC parameters can not retrieve the phase information, which is necessary to classify $M$-ray PSK and MSK signals. Timefrequency based features have shown good performance on retrieving the phase information from the received signal. Methods based on Wigner-Ville distribution (WVD) and Cross Margenau-Hill distribution (CMHD) are quite useful [9]. For an online classification system computation complexity and processing time are two essential issues. The SSC parameters along with two time-frequency based features have been chosen because of the calculation simplicity.

\section{SYSTEM MODEL}

In post-disaster scenario, rescue teams start working in the whole area. So, spectrum sensing is needed to detect the presence of rescue teams in the area. W-DAEN [1] will collect 


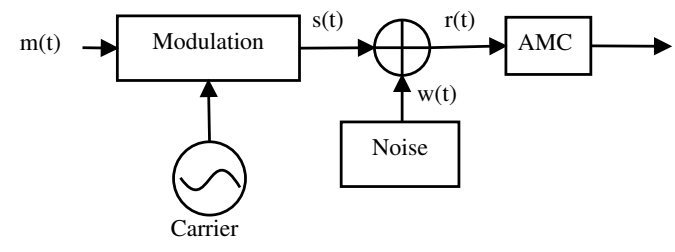

Fig. 1. Simulation model

signal samples from occupied channels and forward to the processing block for modulation classification. Architecture and implementation of the sensor network is beyond the scope of this paper. In this study a simulation has been carried out for the modulation classification system. Information signal $m(t)$ is modulated with a carrier signal $c(t)$. The effect of the environment i.e fading, attenuation etc. degrades the received signal power. Moreover, the noise generated by the receiver hardware makes it worse. For the narrow-band signal the dynamic effect of wireless channel is discarded, instead a slow fading channel is assumed. So the signal to noise ratio (SNR) due to the receiver thermal noise is a sufficient parameter to check the classification performance. For simplicity the whole receiver architecture is not developed in the simulation. The intermediate frequency (IF) signals were generated for different modulation schemes and fed directly to the AMC unit. Block diagram of the system model is presented in fig. 1.

The formulation used to generate the modulated signals are presented here. The received signal can be represented by

$$
r(t)=s(t)+w(t)
$$

where $s(t)$ is the modulated signal and the $w(t)$ is gaussian noise.

The signal $s(t)$ varies with different modulation schemes. For example in case of analog modulations

$$
\begin{gathered}
s_{\mathrm{AM}}(t)=[1+k m(t)] \cos \left(2 \pi f_{\mathrm{c}} t\right) \\
s_{\mathrm{FM}}(t)=\cos \left[2 \pi f_{\mathrm{c}} t+k_{\mathrm{f}} \int_{\infty}^{t} m(\tau) d \tau\right]
\end{gathered}
$$

where, $k$ and $k_{\mathrm{f}}$ are the modulation index for $\mathrm{AM}$ and FM respectively. $f_{\mathrm{c}}$ denotes the carrier frequency. $m(t)$ is a real speech signal. The difference between these two schemes can be checked by simply finding the amplitude and frequency variations. Modulated signal $s(t)$ can be written in a more generic form as

$$
s(t)=I_{m}(t) \cos \left(2 \pi f_{\mathrm{c}} t\right)-Q_{m}(t) \sin \left(2 \pi f_{\mathrm{c}} t\right)
$$

here $I_{m}(t)$ and $Q_{m}(t)$ are "in-phase" and "quadrature" components of the message signal $m(t)$ respectively. For digital modulations the information signal $m_{\mathrm{d}}(t)$ can be represented by

$$
m_{\mathrm{d}}(t)=\sum_{i} \gamma_{i} g(t-i T)
$$

Here, $\gamma_{i}$ is the symbol value at time $i, T$ is symbol duration and $g$ is shaping pulse with window width $T$. In case of ASK

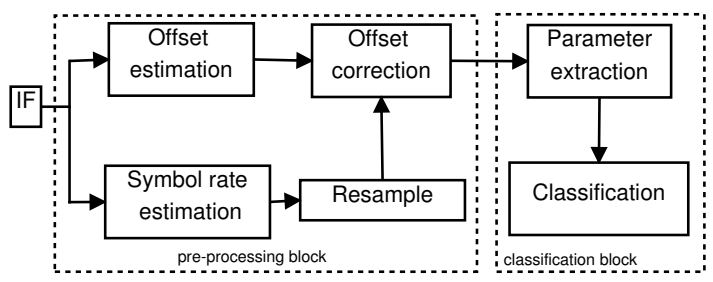

Fig. 2. Data processing steps

signals the quadrature component of $s(t)$ in equation 4 is zero, and can be written as:

$$
s_{\mathrm{ASK}}(t)=\sum_{i} \alpha_{x} g(t-i T) \cos \left(2 \pi f_{\mathrm{c}} t+\psi_{\mathrm{c}}\right)
$$

where, $\left\{\alpha_{i} \in(0,1)\right\}$, and $\psi_{\mathrm{c}}$ is carrier phase that may vary in dynamic channel environment and introduce non-zero quadrature component. For FSK signals

$$
s_{\mathrm{FSK}}(t)=\cos \left(2 \pi f_{\mathrm{c}} t+\psi(t)\right)
$$

here, $\psi(t)$ is the integral over time of the message signal $m_{\mathrm{d}}(t)$. So these two signals can also be separated by looking at the amplitude and frequency variations. In PSK signals the amplitude in the symbol duration becomes constant but the "in phase" and "quadrature" signals are dependent.

$$
s_{\mathrm{PSK}}(t)=\sum_{i} g(t-i T) \cos \left(2 \pi f_{\mathrm{c}} t+\psi_{i}\right)
$$

Here, the symbol $\psi_{i}$ takes the values $\left(0+\left(2 m_{i}+1\right) \frac{\pi}{M}\right)$ with $0 \leq m_{i} \leq(M-1)$ and $M$ is the modulation level. For BPSK and QPSK the value of $M$ is 2 and 4 respectively. In this study following assumptions are made for the received signal

1) The received signals are narrowband and real.

2) The $s(t)$ received by the modulation classifier is already down-converted to a known intermediate frequency(IF) with an unknown offset.

3) The message signal is independent and identically distributed (i.i.d.)

4) Modulation types are AM, FM, 2ASK, 2FSK, BPSK, QPSK, $\pi / 4$ QPSK and MSK

\section{Modulation ClassificATION}

To mitigate the effect of RF impairments from the IF signal some pre-processing is necessary before the feature extraction. Compensation for frequency offset and symbol timing error should be done. Figure 2 shows the flow of signal processing in the considered system.

\section{A. Carrier and symbol rate estimation}

The carrier offset occurs due to the modulation frequency mismatch between the transmitter and receiver. Discrete Fourier transform (DFT) and Fast Fourier transform (FFT) based approaches are widely used to estimate the frequencies in a signal. But these processing requires a prior knowledge of the symbol rate. Data aided (DA) techniques where known pilot symbols are transfered periodically to align with the 
carrier [10] are widely used in communication systems. But in modulation classification environment no communication with the emergency emitters make the DA infeasible. Non data aided (NDA) methods are developed to overcome this problem. Correlation and cyclostationarity based approaches [9], [11], [12] are becoming popular for this purpose. A correlation based approach is considered here. Signal generated by the transmitter with a carrier $f_{\mathrm{c}}$ is

$$
s(t)=I_{\mathrm{s}}(t) \cos \left(2 \pi f_{c} t\right)-Q_{\mathrm{s}}(t) \sin \left(2 \pi f_{c} t\right)
$$

So, the following "in-phase" and "quadrature" signals are extracted by the receiver

$$
\begin{aligned}
I_{\mathrm{r}}(t) & =I_{\mathrm{s}}(t) \cos \left(2 \pi\left(f_{\mathrm{c}}-f_{\mathrm{rc}}\right) t\right)-Q_{\mathrm{s}}(t) \sin \left(2 \pi\left(f_{\mathrm{c}}-f_{\mathrm{rc}}\right) t\right) \\
Q_{\mathrm{r}}(t) & =I_{\mathrm{s}}(t) \sin \left(2 \pi\left(f_{\mathrm{c}}-f_{\mathrm{rc}}\right) t\right)+Q_{\mathrm{s}}(t) \cos \left(2 \pi\left(f_{\mathrm{c}}-f_{\mathrm{rc}}\right) t\right)
\end{aligned}
$$

Here, $f_{\mathrm{rc}}$ is the receiver frequency. Hence, the received complex envelope becomes,

$$
r(t)=I_{\mathrm{r}}(t)+i Q_{\mathrm{r}}(t)=s(t) e^{2 j \pi\left(f_{\mathrm{c}}-f_{\mathrm{rc}}\right) t}
$$

In ideal case, $f_{\mathrm{c}}=f_{\mathrm{rc}}$, so $r(t)=s(t)$. But in reality $f_{\mathrm{c}}=$ $f_{\mathrm{rc}}+\Delta f$. Now, the received signal becomes,

$$
r(t)=s(t) e^{2 i \pi \Delta f t}
$$

For an $M$-ray PSK signal, the $s(t)$ can be expressed as equation 5 So, the received signal becomes

$$
r(t)=\sum_{i} \gamma_{i} h(t-i T) e^{-2 j \pi \Delta f t}+w(t)
$$

Where, $h(t)$ is the convolution of the $g(t)$ and the channel impulse response with the awgn $w(t)$. The non-conjugate autocorrelation of the squared received signal $R\left\{r(t)^{2}\right\}$ can be written as

$$
R\left\{r(t)^{2}\right\}=R\left\{\gamma_{i}^{M}\right\} \sum_{i}(h(t-i T))^{2} e^{-4 i \pi \Delta f t}
$$

This function can be represented by the Fourier series with the major coefficient at the frequency $M(\Delta f)$. Here, $M$ is the modulation level. So the frequency offset can be calculated by finding the maxima of the fourier transform of the $r^{M}(t)$. To increase the accuracy of carrier offset estimation, the received signal sequence has been divided into some segments. And the offset is calculated for each segment. Mean of these values are taken as the final CFO.

$$
\Delta \hat{f}=\frac{1}{N_{s}} \sum_{i=1}^{N_{s}} \Delta f_{i}
$$

here, $N_{s}$ is the number of signal segments. The signal $r(t)$ is then corrected by again modulating with a signal of $\Delta \hat{f}$ frequency. The bandwidth is simply calculated from the signals spectrum obtained from the filter. For the digital modulated signals the symbol rate estimation is an important parameter for time-frequency analysis. Moreover the calculation of oversample rate is necessary for the fifth and sixth features. The algorithm discussed in [13] has been adopted here. The steps to estimate the symbol rate are

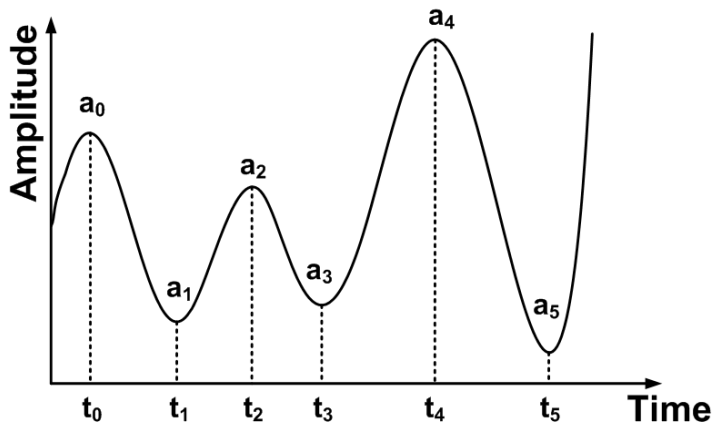

Fig. 3. SSC amplitude and period

- Calculate the Hilbert transform of $r(t)$

- Find FFT of squared envelope with M points and find the maximum $n$ of the absolute value.

- Then estimate the symbol rate as $R_{s}=\left|n . f_{s} / M\right| . f_{s}$ is the rough sampling rate.

\section{B. Feature extraction}

A total of six parameters are used to classify eight modulation schemes. In SSC waveforms are characterized as a function of their relative amplitudes and phases by exploiting the fact that a signal consists of a set of consecutive segments. Each segment has amplitude and period characteristics that are unique for particular frequency combinations and statistically well-behaved [8] i.e. the mean and variance of the segments are consistent. One pre condition of such analysis is the proper, adequate and accurate sampling of the received signal. As mean and variance of the segments are consistent for a certain type of waveform these characterization can be used to classify the received signal. Four SSC parameters are amplitude mean, period mean, amplitude deviation and period deviation. These parameters can be observed from Fig. 3 . The waveform is a combination of multiple frequencies represented by a series of extrema. SSC segments are defined by the area bounded by two consecutive extrema (maxima and minima). A waveform with ' $N$ ' extrema ( $N / 2$ Maxima $+N / 2$ Minima) has ' $N-1$ ' $\mathrm{SSC}$ segments. In the figure six extrema produces five SSC segments. Extrema amplitude and periods are denoted as $\left(a_{0}-a_{5}\right)$ and $\left(t_{0}-t_{5}\right)$ respectively. However, the detection of the extrema is sensitive to the carrier frequency estimation. Mathematically, the segment amplitudes and periods are calculated by following equations in (16).

$$
\begin{gathered}
A_{i}=\left|a_{i}-a_{i-1}\right| \\
T_{i}=\left|t_{i}-t_{i-1}\right|
\end{gathered}
$$

where,

$A_{i}, T_{i}=$ Amplitude and period of the $i$-th segment,

$a_{i}, t_{i}=$ Amplitude and period at the concluding extremum of the segment, and

$a_{i-1}, t_{i-1}=$ Amplitude and period at the beginning extremum of the segment respectively. 
These two values are used to calculate first four features by the following four equations in (17).

$$
\begin{gathered}
A_{\mathrm{M}}=\left(\sum_{i=1}^{N_{\mathrm{S}}} A_{i}\right) / N_{\mathrm{S}} ; \quad A_{\mathrm{D}}=\left(\sum_{i=1}^{N_{\mathrm{S}}}\left|A_{i}-A_{\mathrm{M}}\right|\right) / N_{\mathrm{S}} \\
T_{\mathrm{M}}=\left(\sum_{i=1}^{N_{\mathrm{S}}} T_{i}\right) / N_{\mathrm{S}} ; \quad T_{\mathrm{D}}=\left(\sum_{i=1}^{N_{\mathrm{S}}}\left|T_{i}-T_{\mathrm{M}}\right|\right) / N_{\mathrm{S}}
\end{gathered}
$$

here,

$A_{\mathrm{M}}=$ Amplitude mean, $T_{\mathrm{M}}=$ Period mean, $A_{\mathrm{D}}=$ Amplitude deviation, $T_{\mathrm{D}}=$ Period deviation, $N_{\mathrm{S}}=$ Number of SSC segments.

SSC parameters can distinguish among the modulation schemes with amplitude and frequency variations only. To classify different MPSK signals time-frequency based parameters are used [9]. WVD represents good time-frequency resolution. WVD can be written as

$$
W_{\mathrm{s}}(t, f)=\int_{-\infty}^{\infty} S(f+\alpha / 2) S^{*}(f-\alpha / 2) e^{j 2 \pi t \alpha} d \alpha
$$

Here, $S(f)$ is the Fourier transform of $s(t)$ and $*$ means complex conjugate. The instantaneous frequency can be obtained by taking the first moment of WVD with respect to the frequency.

$$
f_{\mathrm{m}}(t)=\frac{\int_{-\infty}^{\infty} f . W_{s}(t, f) d f}{\int_{-\infty}^{\infty} W_{s}(t, f) d f}
$$

So for signal with variable frequency there will be multiple steps. Therefore the fifth feature is obtained by applying a median filter to equation 19

$$
m_{\mathrm{f}}=\frac{1}{N} \sum_{i=1}^{N}\left|f_{\mathrm{m}}(i)-\frac{1}{N} \sum_{j=1}^{N} f_{\mathrm{m}}(j)\right|
$$

This feature is helpful to distinguish between FSK and MSK signals. CMHD of a signal can preserve the phase information. The CMHD is represented as [14]

$C_{\mathrm{S}}(t, f)=\frac{1}{2} \int_{-\infty}^{\infty}\left\{\left[s(t+\tau)+s(t) e^{j 2 \pi f_{\mathrm{c}} \tau}\right] e^{-j 2 \pi f_{\mathrm{c}} t}\right\} e^{-j 2 \pi f \tau} f \tau$

The CMHDs, show terms that are related to phase or amplitude information of the signal under analysis, in a row along time at the carrier frequency. This row can be extracted from the CMHD as

$$
g(t)=\left|C_{s}\left(t, f=f_{c}\right)\right|
$$

Therefor the sixth feature is calculated by

$$
m_{\mathrm{p}}=\sqrt{\frac{1}{N} \sum_{i=1}^{N} g_{m}^{2}(i)-\left(\frac{1}{N}\left|g_{m}(i)\right|\right)^{2}}
$$

Here, $g_{m}$ is the maximum value of all the received symbols.

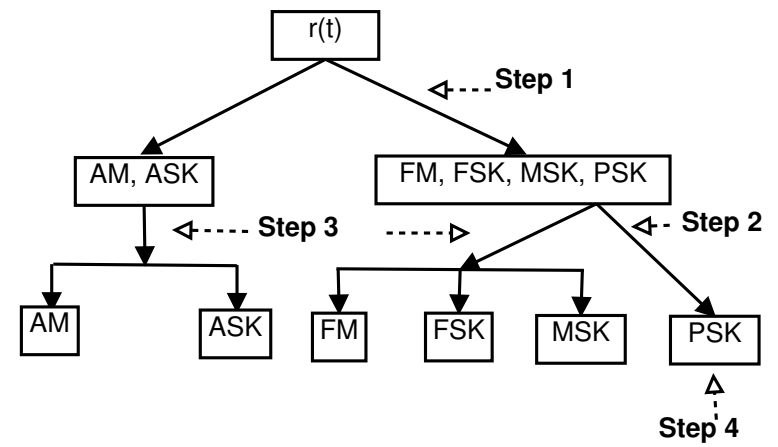

Fig. 4. Decision tree steps

\section{Classification algorithm}

Feature based modulation classification are best suited when the set of target modulation schemes are known apriori. The algorithm implemented here has four steps.

1) Carrier estimation and readjustment

2) SSC and time-frequency based key feature extraction

3) Training the system with known signals

4) Classification of unknown data.

The extracted features are then used for the classifications. For the proposed system the target modulation classes (ana$\log$ and digital) are assumed to be known in advance. The extracted six features are used in four steps for classification.

1) Separate signals with fixed and variable amplitude (using $A_{\mathrm{M}}$ and $\left.A_{\mathrm{D}}\right)$

2) Separate signals with single and multilevel frequencies (using $T_{\mathrm{M}}, T_{\mathrm{D}}$ and $m_{\mathrm{f}}$ ).

3) Check the amplitude and frequency levels to distinguish among analog and digital schemes (SSC features)

4) Separate the PSK schemes by checking phase levels (using $m_{\mathrm{p}}$ )

These steps are presented in figure 4.

As the modulation classes are finite a supervised learning based decision tree classification algorithm can be used here. In this study decision tree algorithm based on C4.5 [15] is used for training set generation and classification purposes. $\tau^{\text {The algorithm is developed in JAVA environment called as }}$ J48 and included in WEKA [16]. J48 decision tree algorithm is relatively faster for numeric data inputs that makes it most suitable for the proposed classification system.

\section{Simulation Results}

A recorded continuous voice signal modulated by AM and FM schemes has been generated as the input signal for the analog modulation. For the digital modulation, PN sequence modulated with 2ASK, 2FSK, BPSK, QPSK, $\pi / 4$ QPSK and MSK scheme have been used as the input signal. A $500 \mathrm{KHz}$ carrier signal has been used with a bandwidth of $250 \mathrm{KHz}$. For this simulation a random frequency deviation from 10 to $20 \mathrm{KHz}$ has been added with the modulated signal. Later the offset is corrected by the CFO estimation. For digital schemes the symbol rate is used as $20 \mathrm{ksps}$. The roll-off factor of 0.5 


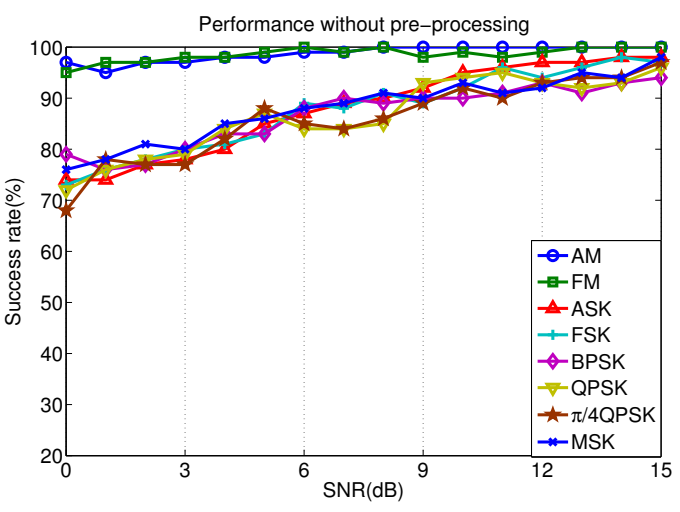

Fig. 5. Classification performance without pre-processing

is used for the raised cosine filter. A frequency deviation of $5 \mathrm{KHz}$ is used for FSK modulation. AWGN of $0-15 \mathrm{~dB}$ have been considered for this simulation.

Received sample streams are divided into 100 segments with 1024 samples in each segment. The classification algorithm is applied to each segment. The final decision is made by the majority rule[2]. At first signals for each target modulation type with SNR 0-15 dB has been generated to train the system. Afterward new signals with different voice data and PN sequence have been generated and feed to the trained system for classification. The performance of the classification is checked for the system with and without the pre-processing block in figure 2. Figure 5 represents the classification performance without any pre-processing. The performance for the digital modulation (especially $M$-ray PSK) schemes has been degraded because of confusion among same type of signals. Figure 6 is showing the results after pre-processing. These results confirm the necessity of preprocessing to improved the classification success rate. In this simulation, success rate is improved by more than $10 \%$. So this study suggests necessity of frequency correction and resampling of the received signal before the feature extraction. Black curves in figure 6 are obtained by using the parameters used by [4]. Seven parameters have been calculated from the generated signals and the performance are obtained by using a decision tree algorithm. The proposed system outperformed these parameters by a big margin also.

\section{CONCLUSION}

A decision tree based modulation classification system has been investigated in this study. Four SSC parameters and two time-frequency based parameters are used for the classifications. The frequency offset correction and symbol rate estimations have improved the classification performance. Effect of symbol timing error on the features and physical implementation are identified as potential future study. The system also considered narrowband signals. Performance of wideband systems for the proposed algorithm is another interesting future study.

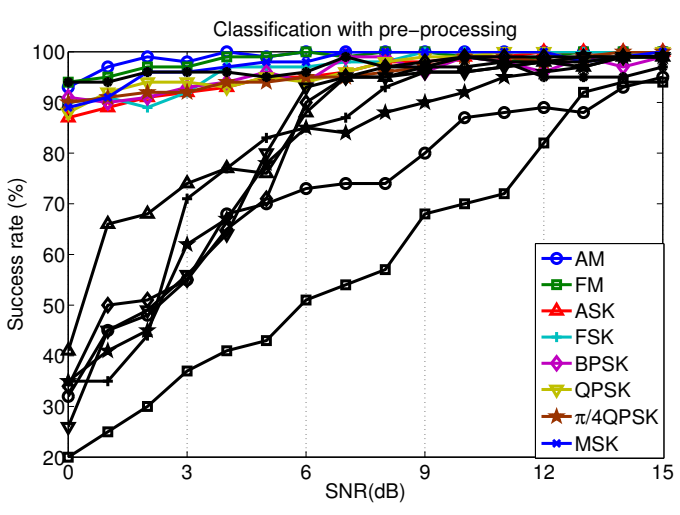

Fig. 6. Classification performance with pre-processing (color) and performance compared with [4] (black)

\section{REFERENCES}

[1] A. Rahman, S. Haniz, A.and Khadka, M. Gahadza, Iswandi, M. Kim, and J. Takada, "Design and implementation of a cognitive radio based emergency sensor network in disaster area," in IEICE Technical Report, no. SR2009-27, 2009.

[2] E. E. Azzouz and A. K. Nandi, Automatic Modulation Recognition of Communication Signals. Kluwer Academic Publishers, 1996.

[3] A. Nandi and E. Azzouz, "Algorithms for automatic modulation recognition of communication signals," Communications, IEEE Transactions on, vol. 46, no. 4, pp. 431 -436, Apr. 1998.

[4] E. Azzouz and A. Nandi, "Procedure for automatic recognition of analogue and digital modulations," Communications, IEE Proceedings, vol. 143, no. 5, pp. 259-266, 1996.

[5] D. Boudreau, C. Dubuc, F. Patenaude, M. Dufour, J. Lodge, and R. Inkol, "A fast automatic modulation recognition algorithm and its implementation in a spectrum monitoring application," in Military Communications Conference Proceedings, vol. 2, pp. 732 -736, 2000.

[6] J. Yang, X. Wang, and $\mathrm{H}$. Wu, "Modified automatic modulation recognition algorithm," in Wireless Communications, Networking and Mobile Computing proceedings, pp. $1-4,2009$.

[7] A. Hossen, F. Al-Wadahi, and J. A. Jervase, "Classification of modulation signals using statistical signal characterization and artificial neural networks," Engineering Applications of Artificial Intelligence, vol. 20, no. 4, pp. $463-472$, 2007. [Online]. Available: http://www.sciencedirect.com/science/article/ B6V2M-4MFK41B-1/2/715d4265be8722e0e887ded982901be3

[8] H. Hirsch, "Statistical signal characterization-new help for real-time processing," in Aerospace and Electronics Conference Proceedings, pp. 121-127, 1992.

[9] Y. Ye and M. Wenbo, "Digital modulation classification using multilayer perceptron and time-frequency features," Journal of Systems Engineering and Electronics, vol. 18, no. 2, pp. 249 - 254, 2007.

[10] W.-Y. Kuo and M. Fitz, "Frequency offset compensation of pilot symbol assisted modulation in frequency flat fading," Communications, IEEE Transactions on, vol. 45, no. 11, pp. 1412 -1416, Nov. 1997.

[11] P. Ciblat, P. Loubaton, E. Serpedin, and G. Giannakis, "Performance analysis of blind carrier frequency offset estimators for noncircular transmissions through frequency-selective channels," Signal Processing, IEEE Transactions on, vol. 50, no. 1, pp. 130 -140, Jan. 2002.

[12] G. Zhou and G. Giannakis, "Harmonics in multiplicative and additive noise: performance analysis of cyclic estimators," Signal Processing, IEEE Transactions on, vol. 43, no. 6, pp. 1445 -1460, Jun. 1995.

[13] X. Tan, H. Zhang, Y. Sheng, and W. Lu, "Blind modulation recognition of psk signals based on constellation reconstruction," in Wireless Communications and Signal Processings, pp. 1 -6, 2010.

[14] H. Ketterer, F. Jondral, and A. Costa, "Classification of modulation modes using time-frequency methods," in Acoustics, Speech, and Signal Processing Proceedings, vol. 5, pp. 2471-2474, 1999.

[15] J. R. Quinlan, C4.5: Programs for Machine Learning. Morgan Kaufmann, 1993.

[16] WEKA documentation. [Online]. Available: http://www.cs.waikato.ac. $\mathrm{nz} / \mathrm{ml} /$ weka/index_documentation.html 